\title{
Prevalence and Risk Factors Associated with S. haematobium Egg Excretion during the Dry Season, Six Months following Mass Distribution of Praziquantel (PZQ) in 2017 in the Bafia Health Area, South West Region Cameroon: A Cross-Sectional Study
}

\author{
Vicky Daonyle Ndassi $\left(\mathbb{D},{ }^{1}\right.$ Judith Kuoh Anchang-Kimbi $\mathbb{D}^{1},{ }^{1}$ Irene Ule Ngole Sumbele $\mathbb{D}^{\mathrm{D}}{ }^{1}$ \\ Godlove Bunda Wepnje, ${ }^{1}$ and Helen Kuokuo Kimbi $\mathbb{1}^{1,2}$ \\ ${ }^{1}$ Department of Zoology and Animal Physiology, Faculty of Science, University of Buea, P.O. Box 63, Buea, SWR, Cameroon \\ ${ }^{2}$ Department of Medical Laboratory Science, Faculty of Health Sciences, The University of Bamenda, P.O. Box 39, \\ Bambili, NWR, Cameroon \\ Correspondence should be addressed to Vicky Daonyle Ndassi; ndassivicky@gmail.com
}

Received 1 February 2019; Revised 20 April 2019; Accepted 22 May 2019; Published 1 July 2019

Academic Editor: Bernard Marchand

Copyright (C) 2019 Vicky Daonyle Ndassi et al. This is an open access article distributed under the Creative Commons Attribution License, which permits unrestricted use, distribution, and reproduction in any medium, provided the original work is properly cited.

Background. A selective population mass drug administration of PZQ involving school-aged children was carried out in the Bafia Health Area in April 2017. This study investigated the prevalence, intensity, and factors associated with S. haematobium egg excretion in this foci during the dry season, six months after the chemotherapy campaign. Methods. A cross-sectional study including 1001 consenting individuals (aged 3-62 years) was carried out in three localities (Ikata, Bafia, and Munyenge) in the Bafia Health Area between November 2017 and January 2018. Information on sociodemographic, stream usage, and contact behaviour was documented. Schistosoma haematobium ova in urine were detected using membrane filtration technique. Results. The prevalence of S. haematobium egg excretion was $8 \%$ with a higher level recorded in Munyenge (13.2\%) than Ikata (7.5\%) and Bafia (2.8\%). The difference was significant $(\mathrm{p}<0.001)$. Equally, Munyenge had the highest infection intensity $(36.36$ range: $2-200)$ when compared with Ikata (16.25 range: $2-57)$ and Bafia (8.0 range: $0-8)$. Although the age group (5-15 years) was significantly $(\mathrm{p}<0.001)$ associated with more exposure to infested water, this group was less likely (OR: 0.42 95\% CI: 0.19-0.91) associated with S. haematobium egg excretion. The risk of egg excretion increased by 4.79 times (95\% CI: 2.20-10.41) and 3.68 times (95\% CI: 1.59-8.54) among residents in Munyenge and Ikata, respectively. Similarly, frequency to the stream ( $>$ thrice/day) was significantly higher $\left(\chi^{2}=58.73 ; \mathrm{p}<\right.$ 0.001) in Munyenge. Frequent contact (three visits/day) with stream correlated with highest odds of egg excretion (OR: 8.43 95\% CI: 3.71-19.13). Conclusion. The prevalence of S. haematobium egg excretion was low during the dry season. This was most likely attributed to the preventive campaign with PZQ and may parallel low transmission potentials in infested waters during this period.

\section{Introduction}

Worldwide, an estimated 218 million people suffer from schistosomiasis, of whom $\sim 90 \%$ live in Africa [1]. Twothirds of these cases are caused by Schistosoma haematobium, the etiologic agent of UGS [2]. The potential consequences of $S$. haematobium infection include haematuria, dysuria, nutritional deficiencies, lesions of the urinary bladder, hydronephrosis, stunting (in children) [3], and in adults, infertility, cancer, and increased susceptibility to HIV [2, $4,5]$. Mass drug administration with praziquantel is the mainstay of programs for the control of schistosomiasis morbidity. Targeted control on school children is often advocated; however, this strategy usually excludes adults and pregnant women who are potential reservoirs for UGS in endemic communities [6]. There is a growing recognition that treatment alone is not sufficient for effecting elimination. Additional measures such as reducing exposure to infectious 
water, moderating availability of intermediate snail host, and decreasing contamination of water with egg-containing urine or faeces will be required to interrupt transmission $[7,8]$.

A predominant feature of Schistosoma haematobium infection is urinary egg excretion. In the current absence of more sensitive field diagnostics, egg counts by microscopic examination of urine remain the accepted standard method for detecting S. haematobium infection [9-11]. Recent studies indicate that egg excretion exhibits seasonal variation [1214] and may have a parallel presentation with seasonal water contact patterns and snail density fluctuations in the transmission rate of $S$. haematobium $[15,16]$. In some studies, S. haematobium ova excretion in urine have been used as a proxy measure of cercarial infection in intermediate snail host and vector abundance at transmission sites [1618]. The seasonal transmission of S. haematobium varies between countries and even between regions in the same country. In some areas the transmission of S. haematobium is higher in the dry season $[14,19,20]$, while in other areas, it is higher during the rainy season [21, 22]. Also, perennial transmission has been reported in some areas [21, 23]. Praziquantel has been demonstrated to reduce the level of urinary tract morbidity substantially within six months after treatment [23-25]. Nonetheless the sustainability of morbidity reduction will depend on the level of transmission and thus the level of reinfection after treatment [23]. Augusto et al. [26] confirmed that the transmission season has an influence on parasitological cure rate and intensity of $S$. haematobium infection following PZQ administration.

In Cameroon, urogenital schistosomiasis is mesoendemic in the Bafia Health Area [27-29], South West Region. This health area is made up of three rural communities: Ikata, Bafia, and Munyenge. Studies in these various areas revealed high S. haematobium infection rates in school-aged children [30] and pregnant women [29] during the rainy season. Nonetheless, no study has reported the infection level in the dry season. Not only is schistosomiasis influenced by climatic factors, but also human behaviour as well as socioeconomic factors plays a key role in the schistosomiasis transmission process [31-33]. Through various water contacts, the human being ensures the successful transmission of the parasite $[34,35]$. Consistent findings showed that the risk of infection among pregnant women in Munyenge is determined by the frequency and intense contact with infested water during bathing and domestic activities [29, 36]. The communitybased studies carried out in the study did not assess stream contact behaviour in relation to risk of infection. In endemic areas, it is well established that school-aged children are particularly at risk of infection due to high frequency of contact with infested water [37]. Socioeconomic factors such as educational level and marital status may influence attitudes towards water contact in this area $[36,38]$. The first ever community-mass drug administration of PZQ was carried out in the Bafia Health Area in April 2017 with a therapeutic coverage rate of $92.4 \%$. The preventive chemotherapy campaign involved the selective administration of the antischistosomal drug praziquantel to school-aged children [39]. Thus, the present study investigated the prevalence, intensity, and risk factors associated with S. haematobium egg excretion in the entire population of the Bafia Health Area during the dry season, six months following the mass distribution of PZQ.

\section{Methods}

2.1. Study Area. This study was carried out in the Bafia Health Area, an endemic focus located in the Mount Cameroon Area. The Bafia Health Area is comprised of three rural communities: Ikata, Bafia, and Munyenge located between longitudes $9.363 \mathrm{E}$ and $9.292 \mathrm{E}$ and latitudes $4.329 \mathrm{~N}$ and $4.401 \mathrm{~N}$ (Figure 1). The altitude of the area ranges from 87 to $168 \mathrm{~m}$ above sea level. It has a heterogenous population of about 25,018 inhabitants consisting of individuals from several cultural backgrounds including natives from Oroko, Wimbum, Kom, Metta, Ibo, Ngie, Ndop, and Isimbi [29]. The principal occupation of the people is farming, their main cash crops being cocoa and plantains [30].

The Mount Cameroon Area has an equatorial climate with two distinct seasons: a rainy season which lasts from March to October followed by a short dry season of four months (November to February) [40]. The streams in the Bafia Health Area are suitable habitats for the Bulinus species intermediate host and thus constitute the main transmission source of $S$. haematobium infection [36]. The area has a temperature range of about $24-27^{\circ} \mathrm{C}$ which favours a high release and infectivity of cercariae into the waters [30]. The population uses the streams for drinking water, bathing, washing of clothes, and household utensils. These streams are visited by children as young as 2 years and adults as old as 50 years of age or more [30].

In Ikata and Munyenge, the streams are quite close to houses and schools but in Bafia, the lone spring is far from residential areas [28]. The difference in the number of and proximity to streams may account for the difference in prevalence of UGS reported in these areas [28, 29] (Figure 1). Presently, these communities have pipe-borne sources but not all parts of the communities have access to safe water and sometimes water supply is not regular. Thus, the populations still make frequent use of the streams for their daily needs. Nonetheless, following increased piped water sources in Munyenge, S. haematobium infection among pregnant has declined due to reduced stream contact [36].

2.2. Study Participants. The study population included individuals of both sexes aged 3-62 years living in the Bafia Health Area. In order to be included in the study, participants must have lived for at least six months in the study area. The sample size of the study population was calculated considering the UGS prevalence of $34.3 \%$ in the Bafia Health Area reported by Ebai et al. [28]. The sample size was determined using the Lorenz formula [41]. The total number of samples $\mathrm{N}$ is given by $\mathrm{N}=\mathrm{Z}^{2} \mathrm{P}(1-\mathrm{P}) / \mathrm{d}^{2}$ where $\mathrm{Z}$ is the standard normal deviation, $\mathrm{Z}=1.96$ for the confidence level of $95 \%, \mathrm{P}=34.3 \%$ : proportion of UGS prevalence, and $d$ is the total width of the confidence interval (e.g., $0.05= \pm 5$ ). The minimum estimated sample size calculated per locality was 346 . For logistics reasons, 349, 334, and 318 consented participants from Munyenge, Ikata, and Bafia were enrolled into the study, respectively. The sample 


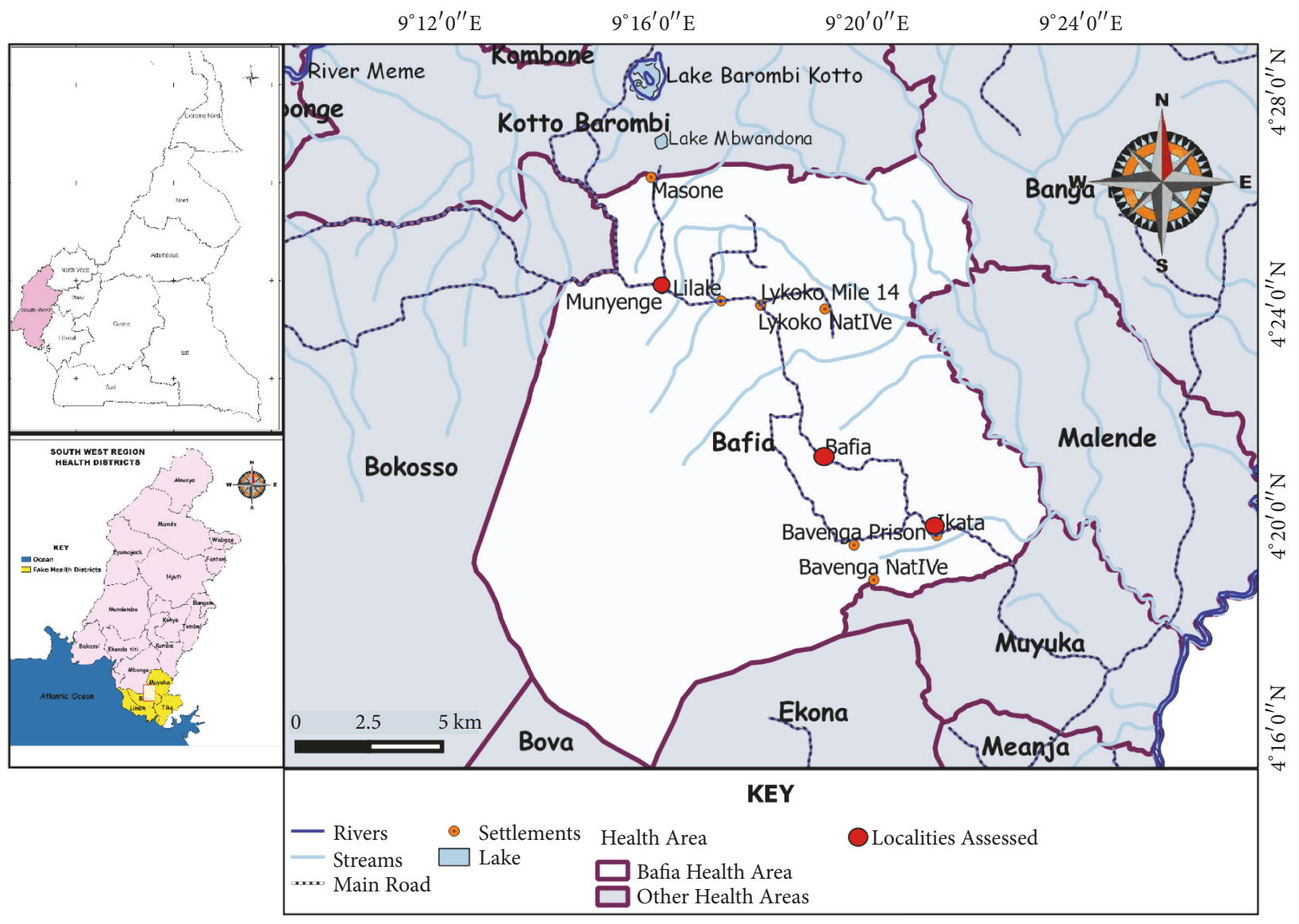

Figure 1: Map showing Bafia Health Area in the Mount Cameroon Area.

size of each locality is within $90-95 \%$ of the expected sample size calculated.

2.3. Study Design. The study was a cross-sectional study carried out from November 2017 to January 2018 involving individuals living in the Bafia Health Area. After obtaining administrative and ethical clearances, an acquaintance visit was made to the chiefs and block heads of the localities concerned in order to inform them about the study procedures. A convenient sampling technique was used to recruit participants into the study. Potential participants were informed about the study in their various localities and only those who met the inclusion criteria and voluntarily gave their consent/assent were enrolled. For children less than 13 years old, assent was gotten from their parents or legal guardians, while for those less than 18 years old, consent was obtained from both the parent and the child. Participants who were 18 years and above gave their consent. The participants were requested to rally at the residence of their respective block heads on the day programmed for sample collection. A semistructured questionnaire was administered to the participants to obtain data on sociodemographic factors and stream contact behaviour. Urine samples were collected and processed for the detection of microhaematuria and $S$. haematobium ova.

2.3.1. Administration of Questionnaire. Participants were interviewed by a field researcher using a semistructured questionnaire to obtain information on demographic (age, sex, and residence), socioeconomic indicators (educational level and occupation), and stream contact behaviour (household water source, number of streams, stream usage, frequency of contact with open water source, and activities carried out in the stream).

2.3.2. Sample Collection and Laboratory Analysis. Each participant who filled the questionnaire was given a sterile, wide mouthed, screw capped plastic bottle carrying their identification number, age, and gender and was properly instructed on how to collect the urine sample between 10 am and $2 \mathrm{pm}$. The younger children (aged $\leq 12$ years) collected their urine with the help of their parents. Immediately after collection, samples were tested for microhaematuria using urine reagent strips (Uripath, Plasmatec Laboratory, UK) (Combi-11) as per manufacturer instructions. Results were expressed as negative or in levels of positivity $(+,++$, or +++$)$ 
excluding presence of traces. All samples were transported on ice bath to the University of Buea, Medical Research Laboratory, and processed within 24 hours of collection.

Schistosoma haematobium ova were identified in urine samples using the filtration technique as described elsewhere [42]. In brief, $10 \mathrm{~mL}$ of urine was filtered using membrane filters (Sterlitech Polycarbonate (PCTE) membrane filters, USA) and the egg count was recorded per $10 \mathrm{mls}$ of urine. The infection intensity was classified as light $(<50$ eggs $/ 10 \mathrm{~mL}$ of urine) or heavy ( $\geq 50$ eggs $/ 10 \mathrm{~mL}$ of urine) as defined by the World Health Organization (WHO) [43]. Microhaematuria was considered as proxy-diagnosis of UGS, an accepted marker in the rapid diagnosis of S. haematobium infection in urine [44]. Thus, an individual was positive for S. haematobium when he/she was diagnosed positive by microscopic examination and/or urine reagent strip.

2.4. Ethical Considerations. The administrative and ethical clearances to conduct the study were obtained from the Regional Delegation of Public Health, South West Region, and Faculty of Health Sciences Institutional Review Board (Ref: 2017/098/UB/SG/IRB/FHS), respectively. Meetings were held with the local authorities of the area to inform them about the study aims, design, benefits, and risks involved and thereafter possible dates and venues scheduled for collection of data and sample. Community health workers were used to inform the communities on prospective study and dates selected for the study. Signed assent and/or consent form indicated voluntary participation. All participants who were positive for UGS were freely treated with praziquantel tablets $(40 \mathrm{mg} / \mathrm{kg}$ of body weight) in collaboration with the local health authorities. Each participant was free to withdraw consent at any time. Identification codes were assigned to each study participant and limited access to study data was maintained to ensure confidentiality.

2.5. Statistical Analysis. The data were analyzed using SPSS version 21.0 (SPSS, Inc., Chicago, IL, USA). Proportions of $S$. haematobium infection were compared between different groups (age groups, sex, area of residence, educational level, occupational status, household water source, stream usage, and frequency to stream) using Pearson Chi-square test. The Kruskal-Wallis test $(\mathrm{H})$ was used to compare the differences in intensities of infections. Odd ratios (OR) and confidence intervals (CIs) were calculated using a Microsoft Excel confidence interval calculator as described by Armitage \& Berry [45] and Newcombe [46]. Variables that had a p-value $<0.20$ in bivariate analysis or explanatory plausibility were included in the multivariate logistic regression model for analysis of risk factors for S. haematobium egg excretion. A p-value of < 0.05 was considered significant.

\section{Results}

3.1. Characteristics of the Study Population. A total of 1001 participants from the three localities were enrolled into the study (Munyenge: 349, Ikata: 334, Bafia: 318), more of them being females. The age of the study participants ranged from 3 to 62 years and only about a third of them had obtained some

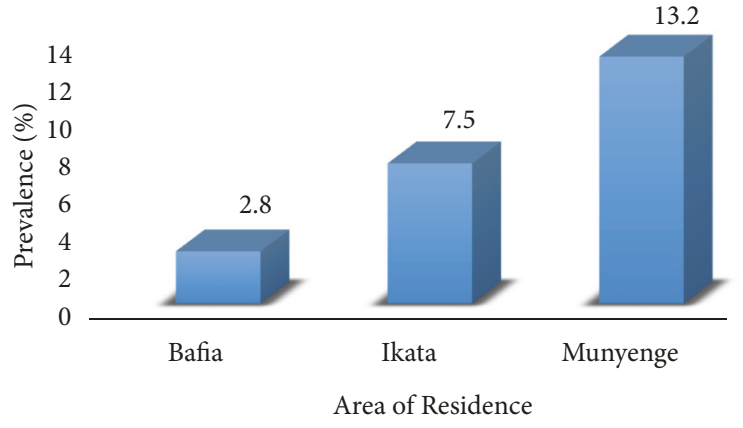

Figure 2: Prevalence of S. haematobium egg excretion in the three localities in the Bafia Health Area.

form of secondary education. A greater percentage of the participants were pupils and farming is a common practice in these communities. Almost all (97.6\%) participants reported stream usage for household chores and/or bathing. Despite presence of some piped water sources in this area, stream usage is predominant; meanwhile the coverage rate for piped water usage as the only source of water is low. The characteristics of the study population are shown in Table 1.

3.2. Prevalence and Intensity of S. haematobium Egg Excretion. Eighty (8\%; 95\% CI: 6-10) of the 1001 participants enrolled were positive for $S$. haematobium infection with varying rates in egg excretion among the three localities (Munyenge (13.2\%), Ikata (7.5\%), and Bafia (2.8\%)) as shown in Figure 2. The difference in prevalence of egg excretion was statistically significant $(\chi 2=24.42, \mathrm{p}<0.001)$. In general, the intensity of egg excretion was low given an overall mean egg load of 33.5 eggs $/ 10 \mathrm{ml}$ of urine (range: $2-200$ ). Although not statistically significant (Kruskal-Wallis test, $\mathrm{H}=2.01, \mathrm{df}=2, \mathrm{p}=0.37$ ), the mean egg load was high in Munyenge (36.36 range: 2-200) (n $=33)$ when compared with Ikata (16.25 range: $2-57(\mathrm{n}=4)$ and Bafia $(8.0)(\mathrm{n}=1)$.

3.3. Risk Factors Associated with S. haematobium Egg Excretion during the Dry Season in the Bafia Health Area. In bivariate analysis, there was an association between $S$. haematobium egg excretion, age $(\mathrm{p}=0.03)$, locality $(\mathrm{p}<0.001)$, and frequency of contact with stream $(\mathrm{p}<0.001)$ (Table 2). Surprisingly, children in age group $<5$ years had more infection (2.54; 95\% CI: 1.04-4.79) when compared with the older age groups. However, the difference was not significant. All individuals who had piped water as the only source of water were diagnosed negative for the infection (Table 2). No association was seen between gender, level of education, occupation, source of water, stream activity, and infection status (Table 2). All three factors associated with infection were retained by multiple regression model analysis (Table 2 ). Gender and educational level have been identified as plausible explanatory variables to the prevalence of schistosomiasis and thus were included in the final model.

The risk of egg excretion increased by 4.79 times (95\% CI: $2.20-10.41)$ and 3.68 times (95\% CI: 1.59-8.54) among residents in Munyenge and Ikata, respectively. It worth noting 
TABLE 1: Sociodemographic characteristics of the study participants.

\begin{tabular}{|c|c|c|c|}
\hline Variable & Characteristic & Number examined (n) & Percentage (\%) \\
\hline \multirow{3}{*}{ Area of residence } & Munyenge & 349 & 34.9 \\
\hline & Ikata & 334 & 33.4 \\
\hline & Bafia & 318 & 31.8 \\
\hline \multirow{2}{*}{ Sex } & Female & 568 & 56.7 \\
\hline & Male & 433 & 43.3 \\
\hline \multirow{4}{*}{ Age group (years) } & $<5$ & 86 & 8.6 \\
\hline & $5-15$ & 476 & 47.6 \\
\hline & $16-39$ & 270 & 27.0 \\
\hline & $\geq 40$ & 169 & 16.9 \\
\hline \multirow{2}{*}{ Educational level } & Primary & 671 & 67.0 \\
\hline & Secondary & 330 & 33.0 \\
\hline \multirow{4}{*}{ Occupation } & Farmer & 356 & 35.6 \\
\hline & Pupil & 436 & 43.6 \\
\hline & Student & 185 & 18.5 \\
\hline & Housewife & 24 & 2.4 \\
\hline \multirow{2}{*}{ Stream usage } & Yes & 977 & 97.6 \\
\hline & No & 24 & 2.4 \\
\hline \multirow{3}{*}{ Source of water } & Stream only & 637 & 63.6 \\
\hline & Stream and piped borne water & 340 & 34.0 \\
\hline & Piped only & 24 & 2.4 \\
\hline \multirow{3}{*}{ Stream activity } & Bathing only & 126 & 12.9 \\
\hline & House chores only & 362 & 37.05 \\
\hline & House chores and bathing & 489 & 50.05 \\
\hline \multirow{5}{*}{ Frequency to stream/daily } & Once & 303 & 31.01 \\
\hline & Twice & 272 & 27.84 \\
\hline & Thrice & 192 & 19.65 \\
\hline & $>$ Thrice & 139 & 14.23 \\
\hline & Hardly & 71 & 7.27 \\
\hline
\end{tabular}

that there was a statistical difference $\left(\chi^{2}=58.73 ; \mathrm{p}<0.001\right)$ between locality and frequency to the stream where people living in Munyenge (20\%; 70/139) frequent (> thrice/day) the stream more than those from Ikata $(11.9 \% ; 37 / 139)$ and Bafia $(10.1 \%$; 32/139). More so, frequent contact with stream correlated with higher odds of egg excretion where more than three visits/days to the stream were associated with highest odds of egg excretion (OR: 8.43 95\% CI: 3.71-19.13) (Table 2). The age group (5-15 years) was less likely (OR: 0.42 95\% CI: 0.19-0.91) associated with $S$. haematobium egg excretion.

3.4. Association between Age, Sex, and Stream Contact Behaviour. We assessed the level of exposure to infested water among the different age and sex groups. The age group (5 -15 years) was significantly associated with more exposure to infested streams. Stream usage $\left(\chi^{2}=19.97 ; \mathrm{p}<0.001\right)$, intense (both domestic chores and bathing) $\left(\chi^{2}=48.98\right.$; $\mathrm{p}<$ $0.001)$, and frequent ( $>$ thrice/week) contact $\left(\chi^{2}=202.88 ; \mathrm{p}<\right.$ 0.001 ) with water was higher among children between 5 and 15 years than any other age group (Table 3 ). Bathing activity only was observed more among the age group $<5$ years; meanwhile the older age group ( $>15$ years) visited the stream frequently for domestic chores only. Type of stream activity was gender-related where bathing was more associated with males whereas domestic chores were carried out more by females. The difference was significant $\left(\chi^{2}=20.84 ; \mathrm{p}<0.001\right)$ (Table 3).

\section{Discussion}

Urogenital schistosomiasis is meso-hyperendemic in the Bafia Health Area and thus a major public health concern in the South West Region of Cameroon. As the streams are the only source of permanent water supply for the community, it is exceedingly difficult to prevent the community from contacting this essential source of water on a daily basis for various uses (bathing; other domestic uses) [27-30]. Given that the first ever community-mass drug administration of PZQ was carried out in the Bafia Health Area in April 2017 to treat and prevent S. haematobium infection among schoolaged children, this study reports on the prevalence, intensity, and risk factors associated with $S$. haematobium egg excretion in the area during the dry season, six months following the mass drug campaign.

In the Bafia Health Area, the prevalence of S. haematobium egg excretion during the dry season was $8 \%$. This 
TABLE 2: Risk factors associated with S. haematobium egg excretion during the dry season.

\begin{tabular}{|c|c|c|c|c|c|}
\hline Variable & Category & $\begin{array}{l}\text { S. haematobium } \\
\text { Positive \% (n) }\end{array}$ & $\begin{array}{c}\text { Unadjusted OR } \\
(95 \% \mathrm{CI})\end{array}$ & $\begin{array}{c}\text { Adjusted OR } \\
(95 \% \mathrm{CI})\end{array}$ & $\mathrm{P}$ - value \\
\hline \multirow{4}{*}{ Area of residence } & Munyenge & $13.2(46)$ & $5.21(2.51-10.83)$ & $4.79(2.20-10.4)$ & $<0.001$ \\
\hline & Ikata & $7.5(25)$ & $2.78(1.27-6.05)$ & $3.68(1.59-8.54)$ & 0.002 \\
\hline & Bafia & $2.8(9)$ & REF & REF & \\
\hline & $\chi^{2} ; \mathrm{P}$ value & $24.416 ;<0.001$ & & & \\
\hline \multirow{3}{*}{ Sex } & Male & $8.8(38)$ & $1.21(0.76-1.91)$ & NA & \\
\hline & Female & $7.4(42)$ & REF & & \\
\hline & $\chi^{2}$; P value & $0.638 ; 0.425$ & & & \\
\hline \multirow{5}{*}{ Age group(years) } & $<5$ & $16.3(14)$ & $2.54(1.12-5.77)$ & $2.0(0.81-4.94)$ & 0.13 \\
\hline & $5-15$ & $6.9(33)$ & $0.97(0.49-1.93)$ & $0.42(0.19-0.91)$ & 0.028 \\
\hline & $16-39$ & $7.8(21)$ & $1.20(0.58-2.52)$ & $0.71(0.32-1.60)$ & 0.41 \\
\hline & $\geq 40$ & $7.1(12)$ & REF & REF & \\
\hline & $\chi^{2}$; P value & $8.958 ; 0.030$ & & & \\
\hline \multirow{3}{*}{ Educational level } & Primary & $9.1(61)$ & $1.64(0.96-2.79)$ & $1.59(0.88-2.85)$ & 0.124 \\
\hline & Secondary & $5.8(19)$ & REF & REF & \\
\hline & $\chi^{2} ; \mathrm{P}$ value & $3.492 ; 0.322$ & & & \\
\hline \multirow{5}{*}{ Occupation } & Farming & $7.9(28)$ & $1.23(0.61-2.48)$ & NA & \\
\hline & Pupil & $9.2(40)$ & $1.46(0.74-2.84)$ & & \\
\hline & Student & $6.5(12)$ & REF & & \\
\hline & Housewife & $0(0)$ & --------- & & \\
\hline & $\chi^{2}$; P value & $5.48 ; 0.360$ & & & \\
\hline \multirow{4}{*}{ Source of water } & Stream only & $8.2(52)$ & $0.99(0.61-1.60)$ & $1.1(0.30-3.72)$ & 0.92 \\
\hline & Stream and piped water & $8.2(28)$ & REF & REF & \\
\hline & Piped water only & $0(0)$ & --------- & --------- & ----- \\
\hline & $\chi^{2} ;$ P value & $2.137 ; 0.343$ & & & \\
\hline \multirow{4}{*}{ Stream activity } & House chores and bathing & $8.0(39)$ & $1.28(0.58-2.81)$ & $0.62(0.27-1.47)$ & 0.28 \\
\hline & House chores only & $9.1(33)$ & $1.48(0.66-3.29)$ & $1.03(0.42-2.51)$ & 0.95 \\
\hline & Bathing only & $6.3(8)$ & REF & REF & \\
\hline & $\chi^{2}$; P value & $1.011 ; 0.603$ & & & \\
\hline \multirow{6}{*}{ Frequency to stream ++ per day } & More than thrice & $18.0(25)$ & $5.82(2.77-12.21)$ & $8.43(3.71-19.13)$ & $<0.001$ \\
\hline & Thrice & $9.9(19)$ & $2.91(1.35-6.27)$ & $4.48(1.93-10.41)$ & $<0.001$ \\
\hline & Twice & $7.7(21)$ & $2.22(1.05-4.69)$ & $3.63(1.64-8.01)$ & 0.001 \\
\hline & Hardly & $5.6(4)$ & $1.58(0.49-5.13)$ & $1.07(0.31-3.72)$ & 0.92 \\
\hline & Once & $3.6(11)$ & REF & REF & \\
\hline & $\chi^{2} ; \mathrm{P}$ value & $27.23 ;<0.001$ & & & \\
\hline
\end{tabular}

$\chi^{2}=$ Pearson Chi-square test; $\mathrm{OR}=$ odd ratio; $\mathrm{aOR}=$ adjusted $\mathrm{OR}$ using multivariate regression analysis.

prevalence is considerably low than the levels reported in the same area during the rainy season by previous authors. Ntonifor et al. [30] reported a prevalence of $40.27 \%$ in Munyenge, while a prevalence of $34.3 \%$ was reported by Ebai et al. [28] in the Ikata-Likoko area. The low prevalence of $S$. haematobium in the present study is most likely attributed to the preventive chemotherapy campaign carried out in the Bafia Health Area. Drug-enhanced protective immune mechanisms against the infection may account for a greater reduction in egg output observed during the dry season. It is well established that PZQ kills mainly the mature worms [47, 48] and is less effective against juvenile (2- to 4 -week-old) parasites [48, 49]. The cumulative deaths of adult $S$. haematobium worms provide the main source of protective antigen that boost antibody responses associated with protection against reinfection [50] as well as reduction in S. haematobium fecundity [51]. However, the presence of infection after PZQ treatment may be due to infection just prior to treatment, reinfection after treatment, or treatment failure. In high transmission areas, the removal of adult worms by treatment may result in low cure rates due to the development of immature worms into egg-producing adults by the time of the follow-up assessment of cure [48]. The low prevalence of egg excretion recorded in the human host during the dry season may as well parallel low transmission potentials at the water contact sites during this period. Ivoke et al. [18] suggested that the community urinary egg output may be a determinant of the infection rate of the snail 
TABLE 3: Association between age, gender, and stream contact behavior.

\begin{tabular}{|c|c|c|c|c|c|c|}
\hline \multirow{2}{*}{$\begin{array}{l}\text { Stream contact behaviour } \\
\text { Stream usage }\end{array}$} & \multicolumn{4}{|c|}{ Age group (years) \% (n) } & \multicolumn{2}{|c|}{ Gender \% (n) } \\
\hline & $<5$ & $5-15$ & $16-39$ & $\geq 40$ & Female & Male \\
\hline Yes & $93.0(80)$ & $99.6(474)$ & $97.0(262)$ & $95.3(161)$ & 98.1(558) & $97.0(419)$ \\
\hline No & $7.0(6)$ & $0.4(2)$ & $3.0(8)$ & $4.7(8)$ & $1.9(11)$ & $3.0(13)$ \\
\hline$\chi^{2} ; \mathrm{P}$ value & \multicolumn{4}{|c|}{$19.970 ;<0.001$} & \multicolumn{2}{|c|}{$1.215 ; 0.301$} \\
\hline \multicolumn{7}{|l|}{ Activity at stream } \\
\hline Bathing & $21.3(17)$ & $15.6(74)$ & 7.3(19) & $9.9(16)$ & $10.4(58)$ & $16.2(68)$ \\
\hline House chores & $37.5(30)$ & $27.6(131)$ & $45.4(119)$ & $50.9(82)$ & $42.8(239)$ & 29.4(123) \\
\hline Both & $41.3(33)$ & $56.8(269)$ & $47.3(124)$ & $39.1(63)$ & $46.8(261)$ & $54.4(228)$ \\
\hline$\chi^{2} ; \mathrm{P}$ value & \multicolumn{4}{|c|}{$48.984 ;<0.001$} & \multicolumn{2}{|c|}{$20.838 ;<0.001$} \\
\hline \multicolumn{7}{|l|}{ Frequency to stream per day } \\
\hline Hardly & $22.5(18)$ & $1.7(8)$ & $3.1(8)$ & $23.0(37)$ & $8.1(45)$ & $6.2(26)$ \\
\hline Once & $47.5(38)$ & $20.7(98)$ & $37.0(97)$ & $43.5(70)$ & $30.6(171)$ & $31.5(132)$ \\
\hline Twice & $17.5(14)$ & $31.9(151)$ & $29.0(76)$ & $19.3(31)$ & $27.6(154)$ & 28.2(118) \\
\hline thrice & $3.8(3)$ & $26.6(126)$ & $17.9(47)$ & $9.9(16)$ & $18.6(104)$ & $21.0(88)$ \\
\hline More than thrice & $8.8(7)$ & $19.2(91)$ & $13.0(34)$ & $4.3(7)$ & $15.1(84)$ & $13.1(55)$ \\
\hline$\chi^{2} ;$ P value & \multicolumn{4}{|c|}{$202.884 ;<0.001$} & \multicolumn{2}{|c|}{$2.528 ; 0.640$} \\
\hline
\end{tabular}

intermediate host as well as the dynamics of intramolluscal larval population at the transmission sites. Nevertheless, the annual transmission pattern for UGS in Bafia Health Area remains to be determined and malacological surveys are imperative in investigating the dynamics of snail host infectivity and cercarial output in this setting.

Residents from Munyenge and Ikata localities were almost five and four times, respectively, more likely at risk of S. haematobium egg excretion than those living in Bafia. In Munyenge, individuals had the tendency to make frequent visits to the stream than those from Ikata and Bafia communities given the presence of more streams and closeness of houses to the streams in this rural community. This results support the well documented observation that people living near the transmission site of schistosomiasis are usually more infected [34, 35]. Similarly, Ebai et al. [28] reported geographical risk associated with infection in the health area. Although not significant, a high intensity of egg excretion was recorded in Munyenge compared with Ikata and Bafia. This may reflect differing transmission intensities among the areas. There is an inverse relationship between PZQ cure rate and intensity of infection in schistosomiasis [52, 53]. It is plausible that children from Bafia had a better cure rate compared with those from more exposed communities.

The prevalence of $S$. haematobium infection recorded among school children contrasts other studies in Cameroon $[30,54]$ as well as other parts of Africa [55], which reported peak prevalence of UGS among school-aged children. In corroboration with the findings of Houmsou [21], in Nigeria, children of preschool age were found to have a higher prevalence of egg excretion $(\mathrm{OR}=2.54)$ than those of the older age groups. The considerable prevalence in this group could be the result of early exposure to infested water bodies when these children are taken along with their mothers or caregivers [21] to water sources for domestic chores, where they often play and swim naked in the infested water [56, 57]. Similarly, bathing/swimming activity was reported more among the youngest age group. Preschoolaged children are usually susceptible to infections due to a weaker immune system [58]. Several studies of UGS tend to focus on school-aged children and adults, with little or no emphasis on preschool children. These neglected groups (pregnant women inclusive) which are believed not to be sufficiently exposed to infection and often left untreated could serve as reservoirs of infection, bringing the distribution of the disease to precontrol level over time [59]. Besides, untreated infected children may develop severe consequences such as malnutrition, reduced ability and impairment of cognitive development which may jeopardise their well-being later in life [4]. In endemic areas, school-aged children are particularly at risk of infection due to high frequency of contact with infested water [37]. Contrary to other studies, our findings revealed that this age group (5-15 years) was less likely associated with egg excretion despite frequent and intense contact with stream. The selective mass distribution of praziquantel in this area involved school-aged children and may explain the decreased infection prevalence observed among this age group. Targeted control of schistosomiasis on school children is often advocated and is usually the main operation in sub-Saharan Africa [59]. Treatment of schistosome infections with praziquantel (PZQ) enhances schistosome-specific immune responses which are associated with protection against reinfection [50] as well as reduction in S. haematobium fecundity [51]. In line with earlier studies carried out in South West Region [29, 30, 60], infection status was comparable between the different sexes although water contact activities were sex-related in this endemic setting. Bathing and domestic chores were associated with the male and female gender, respectively. Studies have reported sex dependent pattern of infection [18]. The lack of association 
between infection and sex may be due to the fact that both activities equally cause the most exposure to cercaria-infested water [61].

It has been observed that water contact patterns are often the major factors with regard to the spatial distribution of S. haematobium infection. Consistent with previous reports in Bafia Health Area, frequent and intense contact with stream were the main factors associated with increased risk of infection $[29,36]$. In this study, community water contact at any point in time is mostly linked to practices including domestic activities and bathing. Leisure water contact particularly during washing of clothes and bathing was the major types of water contact activities significantly associated with school-aged children (5-15 years) in the study area. Laundry, bathing, and recreational swimming are the activities that cause the most exposure to cercaria-infested water because these do involve the immersion of large body parts, for long periods [61]. Recent findings strongly suggest that the extension of more piped water sources in this endemic area will reduce the incidence of infection by reducing the need for intense or frequent contact with infested water [36].

\section{Conclusions}

The prevalence of $S$. haematobium egg excretion is low in the Bafia Health Area during dry season and most likely attributed to the preventive mass chemotherapy campaign with PZQ conducted in the area, six months prior to the study. The low prevalence of egg excretion in the human host may as well parallel low transmission potentials at the water contact sites during this period. The low ova excretion associated with school-aged (5-15 years) children is a likely evidence of the effectiveness of the mass treatment campaign. Since the risk of infection in this area is determined mainly by water contact behaviour, changing the frequency and/or nature of water contact in these communities either through provision of adequate supply of portable water or through health education is a feasible means of preventing and interrupting UGS transmission in the Bafia Health Area.

\section{Abbreviations \\ UGS: Urogenital schistosomiasis \\ OR: Odd ratio \\ CI: Confidence interval \\ SWR: Southwest region \\ PZQ: Praziquantel \\ $\mathrm{H}$ : Kruskal-Wallis test \\ $\chi^{2}$ : Pearson Chi-square test \\ \# OR: Adjusted odd ratio.}

\section{Data Availability}

All datasets on which the conclusions of the research rely are presented in the paper. However, data is available from the corresponding author upon reasonable request.

\section{Conflicts of Interest}

The authors declare that they have no conflicts of interest.

\section{Authors' Contributions}

Vicky Daonyle Ndassi conceived the study, participated in data collection and laboratory analysis, analyzed and interpreted the data, and wrote the manuscript; Judith Kuoh Anchang-Kimbi conceived, designed, and supervised the study, participated in data analysis and interpretation, and contributed to the write-up of the manuscript; Irene Ule Ngole Sumbele participated in the study design, supervision, and revision of the manuscript; Godlove Bunda Wepnje participated in data collection and laboratory analysis; Helen Kuokuo Kimbi participated in the study design, supervision, and revision of the manuscript. All authors read and approved the final manuscript.

\section{Acknowledgments}

The authors appreciate the support and cooperation of the inhabitants, parents, and guardians of the children in the Ikata, Bafia, and Munyenge localities, the health personnel, and laboratory technicians who took part in the study. This work was supported by the special fund for research and modernization given to the authors by the Government of Cameroon.

\section{References}

[1] WHO, "Schistosomiasis," Fact Sheet No. 115, Geneva, World Health Organisation, 2017, http://www.who.int/mediacentre/factsheets/ fs115/en/.

[2] M. J. Van Der Werf, S. J. De Vlas, S. Brooker et al., "Quantification of clinical morbidity associated with schistosome infection in sub-Saharan Africa," Acta Tropica, vol. 86, no. 2-3, pp. 125139,2003

[3] E. Saathoff, A. Olsen, P. Magnussen, J. D. Kvalsvig, W. Becker, and C. C. Appleton, "Patterns of Schistosoma haematobium infection, impact of praziquantel treatment and re-infection after treatment in a cohort of school children from rural KwaZulu-Natal/South Africa," BMC Infectious Diseases, vol. 4, article 40, 2004.

[4] C. H. King and M. Dangerfield-Cha, "The unacknowledged impact of chronic schistosomiasis," Chronic Illness, vol. 4, no. 1, pp. 65-79, 2008.

[5] E. F. Kjetland, P. D. Ndhlovu, E. Gomo et al., "Association between genital schistosomiasis and HIV in rural Zimbabwean women," AIDS, vol. 20, no. 4, pp. 593-600, 2006.

[6] J. F. Friedman, H. K. Kanzaria, and S. T. McGarvey, "Human schistosomiasis and anemia: the relationship and potential mechanisms," Trends in Parasitology, vol. 21, supplementary 8, pp. 386-392, 2005.

[7] W. Evan Secor, "Water-based interventions for schistosomiasis control," Pathogens and Global Health, vol. 108, no. 5, pp. 246254, 2014.

[8] J. E. T. Grimes, D. Croll, W. E. Harrison, J. Utzinger, M. C. Freeman, and M. R. Templeton, "The relationship between water, sanitation and schistosomiasis: a systematic review and 
meta-analysis," PLOS Neglected Tropical Diseases, vol. 8, article e3296, 2014.

[9] K. E. Mott, H. Dixon, E. Osei-Tutu, E. C. England, K. Ekue, and A. Tekle, "Evaluation of the reagent strips in urine tests for detection of Schistosoma haematobium infection: a comparative study in Ghana and Zambia," Bulletin of the World Health Organization, vol. 63, no. 1, pp. 125-133, 1985.

[10] I. A. Eltoum, S. Sulaiman, B. M. Ismail, M. M. M. Ali, M. Elfatih, and M. M. A. Homeida, "Evaluation of haematuria as an indirect screening test for schistosomiasis haematobium: a population-based study in the White Nile Province, Sudan," Acta Tropica, vol. 51, no. 2, pp. 151-157, 1992.

[11] C. Lengeler, H. Mshinda, D. Morona, and D. deSavigny, "Urinary schistosomiasis: testing with urine filtration and reagent sticks for haematuria provides a comparable estimate," Acta Tropica, vol. 53, no. 1, pp. 39-50, 1993.

[12] E. E. Christensen, M. Taylor, S. G. Zulu et al., "Seasonal variations in Schistosoma haematobium egg excretion in schoolage girls in rural KwaZulu-Natal Province, South Africa," South African Medical Journal, vol. 108, no. 4, pp. 352-355, 2018.

[13] G. Augusto, Effect of transmission season on the outcome of treatment of urinary schistosomiasis in school children in Matola and Maputo, Mozambique [Ph.D. thesis], School of Biological and Conservation Sciences, University of KwaZulu-Natal, Durban, South Africa, 2007.

[14] N. G. C. Gbalégba, K. D. Silué, O. Ba et al., "Prevalence and seasonal transmission of Schistosoma haematobium infection among school-aged children in Kaedi town, southern Mauritania," Parasites \& Vectors, vol. 10, article 353, 2017.

[15] H. A. Wilkins and A. Scott, "Variation and stability in schistosoma haematobium egg counts: a four-year study of gambian children," Transactions of the Royal Society of Tropical Medicine and Hygiene, vol. 72, no. 4, pp. 397-404, 1978.

[16] N. J. S. Lwambo, "Transmission of urinary schistosomiasis in Sukumaland, Tanzania. Snail infection rates and incidence of infection in school children," Journal of Helminthology, vol. 62, no. 3, pp. 213-217, 1988.

[17] Z. Xue, M. Gebremichael, R. Ahmad, M. L. Weldu, and A. C. Bagtzoglou, "Impact of temperature and precipitation on propagation of intestinal schistosomiasis in an irrigated region in Ethiopia: suitability of satellite datasets," Tropical Medicine and International Health, vol. 16, no. 9, pp. 1104-1111, 2011.

[18] N. Ivoke, O. N. Ivoke, C. D. Nwani et al., "Prevalence and transmission dynamics of Schistosoma haematobium infection in a rural community of southwestern, Ebonyi State, Nigeria," Tropical Biomedicine, vol. 31, no. 1, pp. 77-88, 2014.

[19] A. A. Nwabueze and K. N. Opara, "Urinary schistosomiasis among school children in riverine communities of delta state, Nigeria: impact of road and bridge construction," Journal of Medical Sciences, vol. 7, no. 4, pp. 572-578, 2007.

[20] K. N. Opara, N. I. Udoidung, and I. G. Ukpong, "Genitourinary schistosomiasis among primary school children in a rural community within the Cross-river basin, Nigeria," Journal of Helminthology, vol. 81, no. 4, pp. 393-397, 2007.

[21] R. S. Houmsou, "Profile of a one year epidemiological study of urinary schistosomiasis in two Local Government Areas (LGAs) of Benue State, Nigeria," Journal of Biomedical Sciences, 2012.

[22] F. Sarkinfada, A.-A. Oyebanji, I. A. Sadiq, and Z. Ilyasu, "Urinary schistosomiasis in the Danjarima community in Kano, Nigeria," The Journal of Infection in Developing Countries, vol. 3 , no. 6, pp. 452-457, 2009.
[23] A. I. Kahama, B. J. Vennervald, Y. Kombe et al., "Parameters associated with Schistosoma haematobium infection before and after chemotherapy in school children from two villages in the Coast province of Kenya," Tropical Medicine \& International Health, vol. 4, no. 5, pp. 335-340, 1999.

[24] C. F. Hatz, B. J. Vennervald, T. Nkulila et al., "Evolution of Schistosoma haematobium - related pathology over 24 months after treatment with praziquantel among school children in southeastern Tanzania," The American Journal of Tropical Medicine and Hygiene, vol. 59, no. 5, pp. 775-781, 1998.

[25] G. Campagne, A. Garba, H. Barkire, C. Vera, and A. Sidiki, "Suivi échographique prolongé d’enfants infestés par Schistosoma haematobium après traitement par praziquantel," Tropical Medicine \& International Health, vol. 6, no. 1, pp. 24-30, 2001.

[26] G. Augusto, P. Magnussen, T. K. Kristensen, C. C. Appleton, and B. J. Vennervald, "The influence of transmission season on parasitological cure rates and intensity of infection after praziquantel treatment of Schistosoma haematobium-infected schoolchildren in Mozambique," Parasitology, vol. 136, no. 13, pp. 1771-1779, 2009.

[27] H. N. Ntonifor, G. N. Mbunkur, and N. W. Ndaleh, "Epidemiological survey of urinary schistosomiasis in some primary schools in a new focus behind mount Cameroon, South West Region, Cameroon," East African Medical Journal, vol. 89, no. 3, pp. 82-88, 2012.

[28] C. B. Ebai, H. K. Kimbi, I. U. N. Sumbele, J. E. Yunga, and L. G. Lehman, "Prevalence and risk factors of urinary schistosomiasis in the ikata-likoko area of Southwest Cameroon," International Journal of Tropical Disease and Health, vol. 17, no. 2, article IJTDH.26669, pp. 1-10, 2016.

[29] J. K. Anchang-Kimbi, D. M. Elad, G. T. Sotoing, and E. A. Achidi, "Co-infection with schistosoma haematobium and plasmodium falciparum and anaemia severity among pregnant women in Munyenge, Mount Cameroon Area: a cross-sectional study," Journal of Parasitology Research, vol. 2017, Article ID 6173465, 12 pages, 2017.

[30] H. N. Ntonifor, A. E. Green, M. O. S. Bopda, and J. T. Tabot, "Epidemiology of urogenital Schistosomiasis and soil transmitted Helminthiasis in a recently established focus behind Mount Cameroon," International Journal of Current Microbiology and Applied Sciences, vol. 4, no. 3, pp. 1056-1066, 2015.

[31] P. Allotey, D. Reidpath, and S. Pokhrel, "Social sciences research in neglected tropical diseases: the ongoing neglect in the neglected tropical diseases," Health Research Policy and Systems, vol. 8 , article 32, 2010.

[32] J. Utzinger, E. K. N'Goran, C. R. Caffrey, and J. Keiser, "From innovation to applications: social ecological context, diagnostics, drugs and intergrated control of schistosomiasis," Acta Tropica, vol. 120, supplementary 1, pp. 121-137, 2011.

[33] A. Gazzinelli, G. Velasquez-Melendez, S. B. Crawford, P. T. LoVerde, R. Correa-Oliveira, and H. Kloos, "Socioeconomic determinants of schistosomiasis in a poor rural area in Brazil," Acta Tropica, vol. 99, no. 2-3, pp. 260-271, 2006.

[34] S. K. Chandiwana, "Community Water contact patterns and the transmission of Schistosoma haematobium in the Highveld regions of Zimbabwe," Social Science and Medicine, vol. 25, no. 5, pp. 495-505, 1987.

[35] J. T. Scott, M. Diakhaté, K. Vereecken et al., "Human water contacts patterns in Schistosoma mansoni epidemic foci in northern Senegal change according to age, sex and place of residence, but are not related to intensity of infection," Tropical Medicine \& International Health, vol. 8, no. 2, pp. 100-108, 2003. 
[36] G. B. Wepnje, J. K. Anchang-Kimbi, V. D. Ndassi, L. G. Lehman, and H. K. Kimbi, "Schistosoma haematobium infection status and its associated risk factors among pregnant women in Munyenge, South West Region, Cameroon following scale-up of communal piped water sources from 2014 to 2017: a crosssectional study," BMC Public Health, vol. 19, article 392, 2019.

[37] L. Worku, D. Damte, M. Endris, H. Tesfa, and M. Aemero, "Schistosoma mansoni infection and associated determinant factors among school children in Sanja Town, northwest Ethiopia," Journal of Parasitology Research, vol. 2014, Article ID 792536, 7 pages, 2014.

[38] H. Sady, H. M. Al-Mekhlafi, M. A. K. Mahdy, Y. A. L. Lim, R. Mahmud, and J. Surin, "Prevalence and associated risk factors of schistosomiasis among children in Yemen: Implications for effective control programs," PLOS Neglected Tropical Diseases, vol. 7, no. 8, pp. 1-10, 2013.

[39] Regional Delegation of Public Health South West Region, Cameroon, Report, 2017.

[40] L. K. Basco, V. F. Ngane, M. Ndounga et al., "Molecular epidemiology of malaria in Cameroon. XXII. Baseline therapeutic efficacy of chloroquine, Amodiaquine and sulfadoxinepyrimethamine in children beforenational drug policy change," The American Journal of Tropical Medicine and Hygiene, vol. 75, pp. 388-395, 2006.

[41] L. Naing, W. Than, and B. Rusli, "Practical issues in calculating the sample size for prevalence studies," Archives of Orofacial Sciences, vol. 1, no. 1, pp. 9-14, 2006.

[42] N. O. Christensen, G. Gotsche, and F. Frandsen, "Parasitological techniques for use in routine laboratory maintainance of schistosomes and used in studies on the epidemiology of human and bovine schistosomiasis, danish bilhaziasis laboratory manual," 1984.

[43] WHO, "Tropical disease research," TDR strategic direction, Schistosomiasis, WHO-TDR, 2002.

[44] WHO, Guidelines for the Evaluation of Soil Transmitted Helminthiasis and Schistosomiasis at Community Level: A Guide for Managers of Control Programme, World Health Organisation, Geneva, Switzerland, 1993.

[45] P. Armitage and G. Berry, Statistical Methods in Medical Research, Blackwell, London, UK, 3rd edition, 1994.

[46] R. G. Newcombe, "Interval estimation for the difference between independent proportions: comparison of eleven methods," Statistics in Medicine, vol. 17, no. 8, pp. 873-890, 1998.

[47] A. A. Sabah, C. Fletcher, G. Webbe, and M. J. Doenhoff, "Schistosoma mansoni: chemotherapy of infections of different ages," Experimental Parasitology, vol. 61, no. 3, pp. 294-303, 1986.

[48] L.-A. T. Tchuenté, D. J. Shaw, L. Polla, D. Cioli, and J. Vercruysse, "Efficacy of praziquantel against Schistosoma haematobium infection in children," The American Journal of Tropical Medicine and Hygiene, vol. 71, pp. 778-782, 2004.

[49] E. K. N'Goran, H. N. Gnaka, M. Tanner, and J. Utzinger, "Efficacy and side-effects of two praziquantel treatments against Schistosoma haematobium infection, among school children from Cote d'Ivoire," Annals of Tropical Medicine and Parasitology, vol. 97, no. 1, pp. 37-51, 2003.

[50] F. Mutapi, P. D. Ndhlovu, P. Hagan et al., "Chemotherapy accelerates the development of acquired immune responses to Schistosoma haematobium infection," The Journal of Infectious Diseases, vol. 178, no. 1, pp. 289-293, 1998.

[51] K. M. Mitchell, F. Mutapi, N. J. Savill, and M. E. J. Woolhouse, "Protective immunity to Schistosoma haematobium infection is primarily an anti-fecundity response stimulated by the death of adult worms," Proceedings of the National Acadamy of Sciences of the United States of America, vol. 109, no. 33, pp. 13347-13352, 2012.

[52] J. Utzinger, E. K. N'Goran, A. N’Dri, C. Lengeler, X. Shuhua, and M. Tanner, "Oral artemether for prevention of Schistosoma mansoni infection: randomised controlled trial," The Lancet, vol. 355, no. 9212, pp. 1320-1325, 2000.

[53] G. Raso, E. K. N'Goran, A. Toty et al., "Efficacy and side effects of praziquantel against Schistosoma mansoni in a community of western Côte d'Ivoire," Transactions of the Royal Society of Tropical Medicine and Hygiene, vol. 98, no. 1, pp. 18-27, 2004.

[54] N. Dakoni, P. Saotoing, R. Tchawe et al., "Epidemiological survey on schistosomiasis caused by Schistosoma haematobium and Schistosoma mansoni in primary schools in the SubDivision of Taibong-Dziguilao, Far-North Region Cameroon," Journal of Applied Biosciences, vol. 90, no. 1, pp. 8397-8407, 2015.

[55] O. A. Babamale, O. H. Kolawole, K. Abdulganiyu, O. A. Abdulkareem, and U. S. Ugbomoiko, "Urogenital schistosomiasis among schoolchildren and the associated risk factors in selected rural communities of kwara state, Nigeria," Journal of Tropical Medicine, vol. 2018, Article ID 6913918, 6 pages, 2018.

[56] A. Garba, N. Barkire, A. Djibo et al., "Schistosomiasis in infants and preschool-aged children: Infection in a single Schistosoma haematobium and a mixed S. haematobium-S. mansoni foci of Niger," Acta Tropica, vol. 115, no. 3, pp. 212-219, 2010.

[57] S. E. Odogwu, N. K. Ramamurthy, N. B. Kabatereine et al., "Schistosoma mansoni in infants (aged $<3$ years) along the Ugandan shoreline of Lake Victoria," Annals of Tropical Medicine and Parasitology, vol. 100, no. 4, pp. 315-326, 2006.

[58] A. K. Simon, G. A. Hollander, and A. H. McMichael, "Evolution of the immune system in humans from infancy to old age," Proceedings of the Royal Society B Biological Science, vol. 282, article 1821, 2015.

[59] O. T. Salawu and A. B. Odaibo, "Maternal schistosomiasis: a growing concern in sub-Saharan Africa," Pathogens and Global Health, vol. 108, no. 6, pp. 263-270, 2014.

[60] H. K. Kimbi, G. B. Wepnje, J. Anchang-Kimbi et al., "Active case detection and prevalence of urinary schistosomiasis and malaria in pupils of kotto barombi, Southwest Cameroon using the CyScope ${ }^{\circledR}$ Fluorescence microscope," International Journal of Tropical Disease and Health, vol. 8, no. 1, pp. 1-12, 2015.

[61] A. O. Omonijo, S. O. Asaolu, and I. E. Ofoezie, "Schistosomiasis transmission and water contact pattern in river Ureje in Adoekiti local government area, Ekiti State," Research Journal of Parasitology, vol. 8, no. 1, pp. 26-36, 2013. 


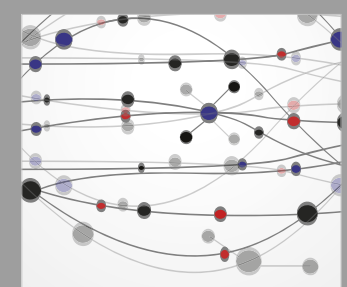

The Scientific World Journal
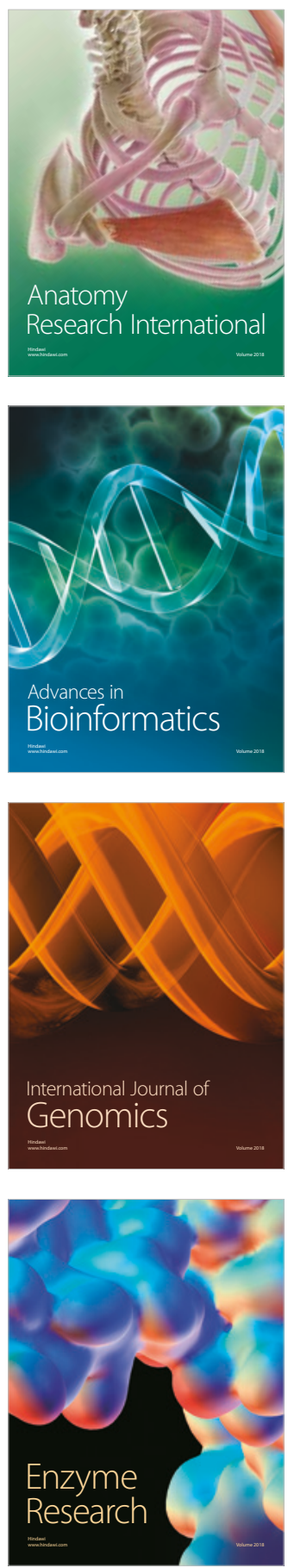
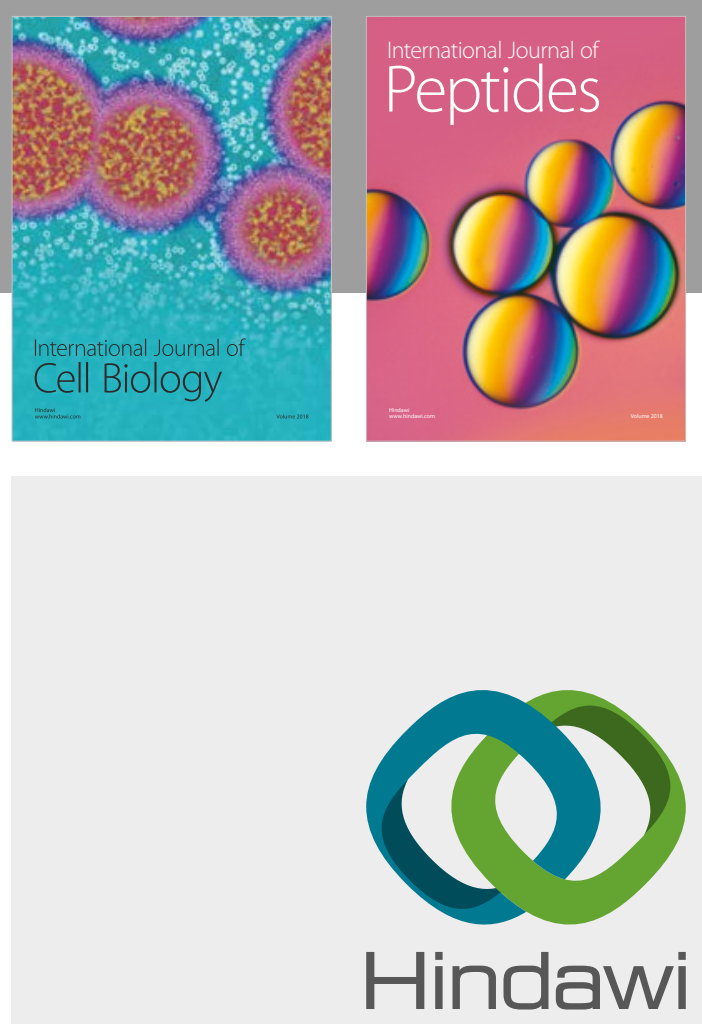

Submit your manuscripts at

www.hindawi.com
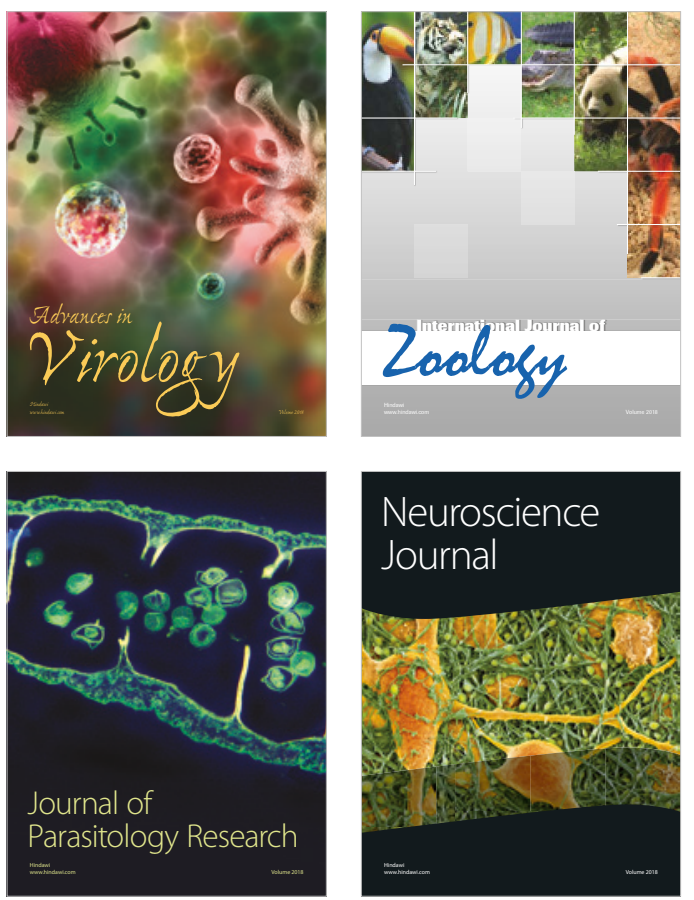
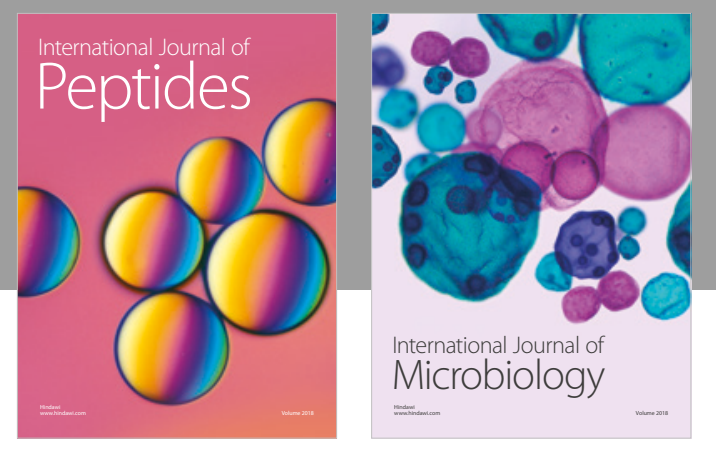

nternational Journal of Microbiology
Journal of
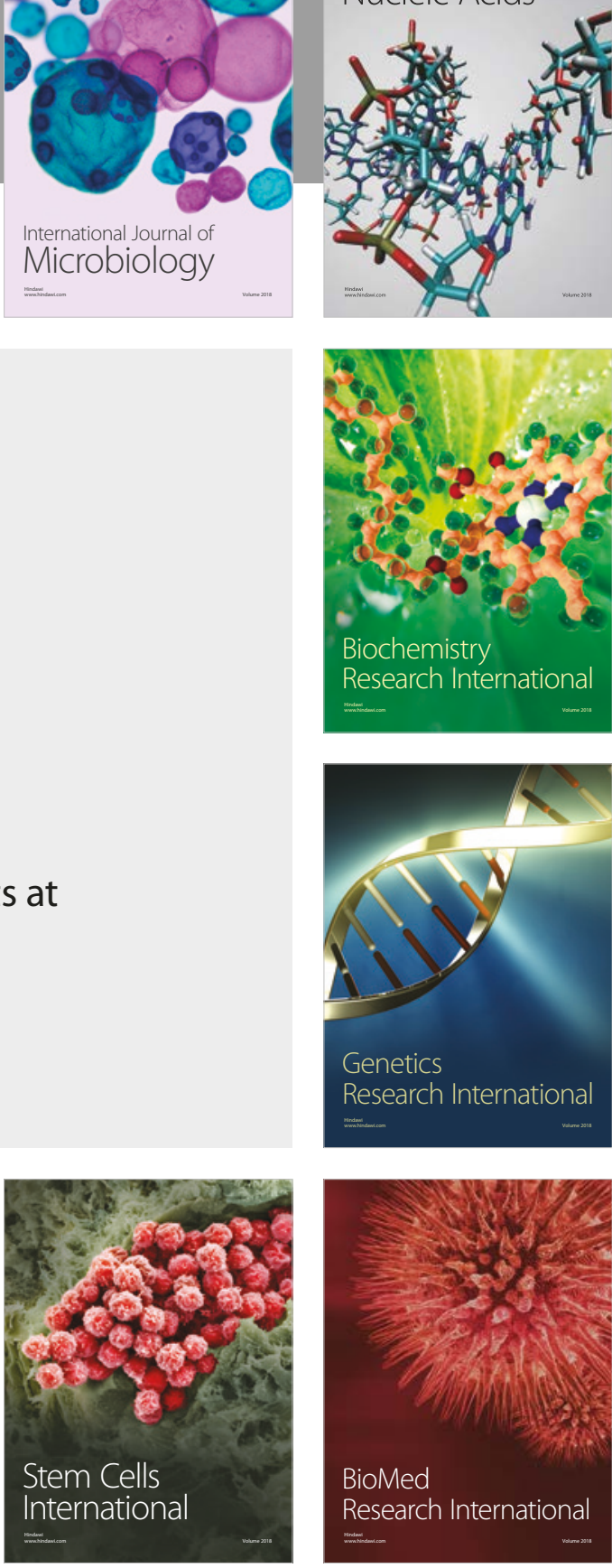
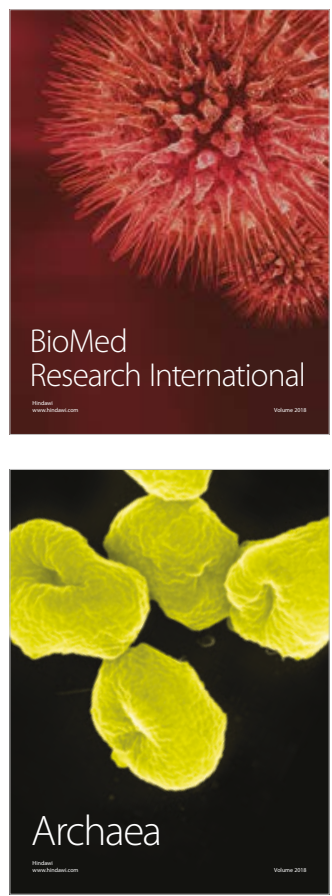